\title{
Use of Regional Anesthesia in Patients with Multiple Sclerosis
}

\author{
Cassandra Gerhart* \\ The University of Kansas Medical Center, 3901 Rainbow Boulevard, Kansas City, KS \\ *Corresponding author: Cassandra Gerhart, RN, BSN, The University of Kansas Medical Center, 3901 Rainbow Boulevard, Kansas City, \\ KS
}

\begin{abstract}
Review Topic: Use of Regional Anesthesia in Patients with Multiple Sclerosis

Background: This article will focus on patients who have traditionally been considered contraindicated for the use of regional anesthesia. Specifically, patients with autoimmune mediated neurologic disorders. The selected pathophysiology of multiple sclerosis and regional anesthesia and their considerations via evaluation of research studies will be presented. Furthermore, suggestions for anesthesia care for these patients will be provided.

Project Aim: The purpose of this article is to and educate anesthesia providers on patients with multiple sclerosis and anesthesia implications for this population. There appears to be a lack of discussion and research in the United States regarding the use of regional anesthesia in these patients. After reading this article, the reader will be able to identify patients at an increased risk of nerve damage, evaluate them properly preoperatively, and objectively determine if the patient is considered a candidate for regional anesthesia.

Data Sources: The research information was gathered via an extensive literature review on Cumulative Index to Nursing and Allied Health Literature (CINAHL), PubMed, and EBSCOhost. Additional resources were also utilized for physiology and pathophysiology purposes: Clinical Anesthesia 7th edition and Nurse Anesthesia $5^{\text {th }}$ edition.
\end{abstract}

Keywords: Multiple sclerosis; Nerve damage; Regional anesthesia; Contraindications; Demyelination; Local anesthetic; Autoimmune

\section{Introduction}

There are several clear benefits of regional anesthesia for surgical procedures. However, patients with pre-existing neurologic disorders create a unique challenge for the anesthesia provider considering the use of regional or neuraxial anesthesia. Specifically, patients with autoimmune mediated neurologic disorders that possess pre-existing neurologic deficits have been thought to be at a higher risk for neural compromise and therefore, are often excluded from the use of regional anesthesia [1]. In all cases where regional anesthesia is considered, the benefits must be weighed against potential complications; the greatest risk being a new or worsened neurologic deficit. With the advancement of regional techniques paired with ultrasound guidance, literature suggests that belonging to this patient population should not necessarily be a contraindication to regional anesthesia [2]. Due to the medicolegal concerns and historic avoidance of regional anesthesia in patients with neurologic disorders, there is a lack of discussion and studies in the United States on this topic. However, increasing support for the use of regional anesthesia for surgical procedures has produced a few studies within the United States and gained even more popularity internationally.

This article will explain the pathophysiology of multiple sclerosis, evaluate current anesthetic practices and recent literature on regional anesthesia for patients with this disorder and provide recommendations for further studies and practice guidelines. After reading this article the reader will be able to properly identify and assess patients at an increased risk for neurologic compromise, 
evaluate the risks and benefits of using regional anesthesia and determine if the patient is a candidate. The reader will also have a better understanding of the pathophysiology of multiple sclerosis and its anesthetic considerations to improve quality of care and perioperative outcomes.

\section{Pathophysiology of Multiple Sclerosis}

Multiple sclerosis (MS) is an inflammatory autoimmune disorder of the central nervous system (CNS). However, recent post-mortem studies have reported some degree of peripheral involvement [3]. Although genetic susceptibility is likely, there is no obvious genetic, environmental or infectious cause that has been identified. Additionally, there is no clear understanding of the immunopathogenesis of the disorder that determines site of tissue damage, variations throughout history, or the severity of disability Pathologically, MS possesses a unique combination of characteristics that include inflammation, demyelination and axonal damage in the CNS; followed by the formation of plaques resulting in further alteration of nerve conduction [4]. The innate immune system is thought to play a major role in the initiation of multiple sclerosis by modification of T and B- lymphocyte function. The adaptive immune system is also involved by activation of CD4 and CD8 cells. It is their polarization to Th1 and Th17 effector cells and cytokine formation that causes damage to the blood brain barrier. This allows autoreactive lymphocytes access to the CNS subsequently leading to the characteristic demyelination associated with the disease [3] (Figure 1).

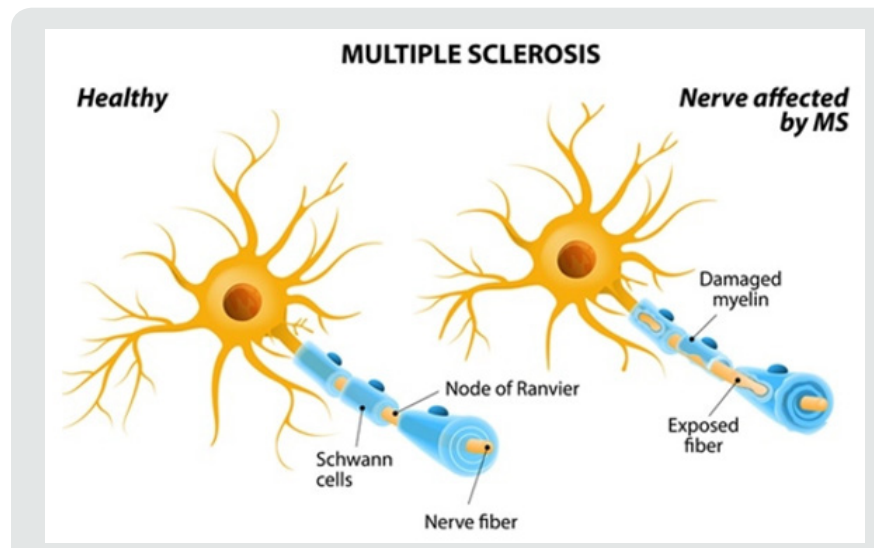

Figure 1: Multiple Sclerosis.

Symptoms associated with MS are often varied with multifocal involvement, but there are two general clinical patternsexacerbating-remitting or chronic progressive. Progression of MS is caused by lack of remyelination and axonal degeneration which is irreversible and causes permanent neurological deficits [3]. Physical manifestations reflect the sites where demyelination has occurred. Although ascending spastic paresis of the skeletal muscles is often prominent, symptoms may also present as sensory deficits, autonomic dysfunction, emotional lability, bladder and bowel dysfunction and visual disturbances. MS exacerbations can be triggered by heat, stress, infections and pregnancy. Diagnosis of MS is made based on clinical features alone or in combination with oligoclonal immunoglobulin irregularities in the cerebrospinal fluid, prolonged latency of evoked potentials, and changes in white matter. There is currently no cure for MS and treatment is directed towards symptom control and slowing the progression of demyelination [4].

\section{Regional Anesthesia Considerations}

Management of anesthesia in patients with MS must include consideration of the impact that surgical stress will have on the natural progression of the disease. Surgical stress may trigger exacerbation of symptoms and an increase in temperature as little as one degree Celsius can result in complete block of conduction of demyelinated nerves. Due to the unpredictable and variable cycles of exacerbation and remission associated with MS, erroneous speculations that there is a relationship between certain drugs or anesthetic technique and exacerbations may be made during the perioperative period. Although there are no known interactions between the drugs used to produce general anesthesia and MS, the use of muscle relaxants can have negative effects on the patient. The use of a depolarizing muscular blocker, such as succinylcholine, is avoided due to the possibility of exaggerated release of potassium in patients with MS. Additionally, when nondepolarizing muscular blockade is used, resistance has been observed which may be explained by the proliferation of extra junctional cholinergic receptors because of upper motor neuron plaques [5]. When appropriate for the procedure, the use of regional anesthesia may allow avoidance of neuromuscular blockade and associated complications. Prior to determining if a patient with MS is a candidate for regional or neuraxial anesthesia, a thorough preoperative assessment must be done. History and pattern of disease, physical and neurological deficits, especially those impacting the respiratory system, oxygen saturation, medications, and previous triggers should be appreciated [4]. Patients with significant respiratory compromise or cognitive dysfunction may notably benefit from the use of regional anesthesia. Additionally, the efficient and prolonged analgesia associated with regional anesthesia may contribute to a less stressful and painful postoperative period decreasing the risk of exacerbations [3].

Peripheral nerve blocks (PNB) are far enough away from central lesions and are considered a safe therapy to use for anesthesia in the patient with MS. However, the addition of epinephrine to a PNB should be avoided to decrease further nerve strain through vasoconstriction. Ultrasound guidance for PNB placement is recommended to decrease risk of mechanical trauma as well as reduce the volume of local anesthetic used to prevent local anesthetic toxicity. Patients with MS are thought to be more sensitive to the effects of local anesthetics, especially when used centrally. Higher concentrations of oligopeptides are 
found in the CSF of patients with MS. Certain oligopeptides block sodium channel activity in the nerves and may be responsible for the profound muscle weakness associated with the disease. Local anaesthetics (LA) share physiologic properties with oligopeptides which may produce a synergistic reaction worsening MS symptom, especially when used for subarachnoid blocks. Therefore, spinal anesthesia should be used with caution. Despite spinal anesthetic concerns, epidural anesthesia is considered a safe alternative because the concentration of LA that approaches the subarachnoid space after an epidural injection is approximately one fourth of the epidurally administered concentration [3]., A main point of concern for anesthesia providers considering the use of regional anesthesia in this patient population is the proposed increased risk that the underlying condition may worsen, or a new deficit will develop. This phenomenon was first described in 1973 as the "double crush phenomenon" by Upton and McComas [1]. It was thought that a nerve with pre-existing neural compromise was more likely than a healthy nerve to incur damage at another point along the nerve. This is known as the double crush phenomenon, described by the photo below [1] (Figure 2).

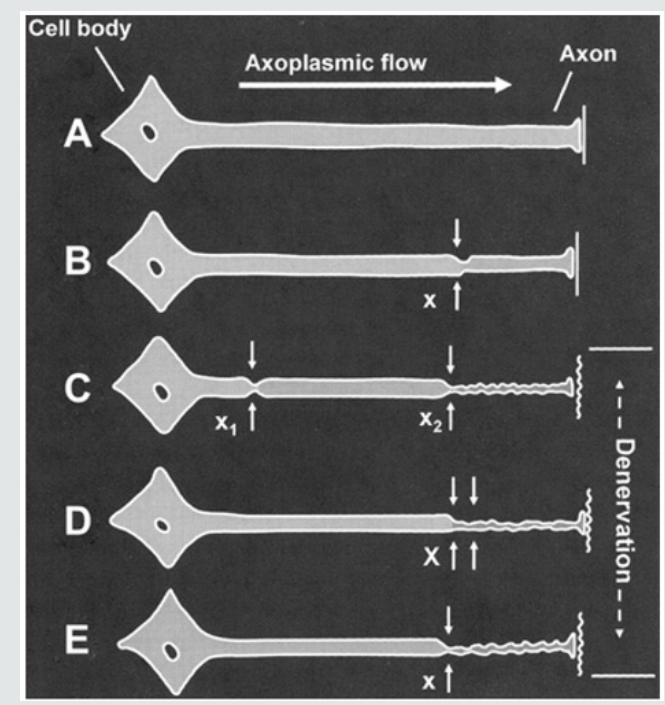

Figure 2: Axoplasmic flow.

The double crush phenomenon refers to the coexistence of at least 2 injury insults or lesions along the same nerve. The theory originally suggested injury was a result from serial nerve compression or restrictions in axonal flow, however it since broadened to include conditions like neurologic disorders and metabolic, toxic or traumatic ischemic insults which may serve as the primary or secondary neural compromise. Patients with pre-existing neurologic disorders present with an assumed first crush as caused by their intrinsic disease thus, theoretically, predisposing them to a second crush. The second crush can be caused by many factors during the perioperative period, including intraoperative trauma or stretch, vascular compromise, hematoma formation, ischemia from tourniquet use or constrictive dressings, or from regional anesthesia related risks such as mechanical trauma from needle placement or LA toxicity [1]. Historically, patients with pre-existing neurological disorders were not offered neuraxial techniques due to fear of worsening neurological deficits. For decades, this has been standard clinical management of these patients because of a recommendation made in 1956 by Dripps and Vandam [6] .This standard of practice was continued because of provider and patient biases as well as the high risk of medicolegal concerns [6]. Despite the fears surrounding double crush phenomenon related to regional and neuraxial anesthesia worsening symptoms in patients with MS, there are several factors such as hyperpyrexia, stress and infection that are known to negatively affect the disease progression or exacerbation [5]. The cause of decline in neurologic function in patients with MS is not clearly understood and may simply be coincidental in the perioperative period regardless of the anesthetic technique used. However, because of the difficulty in establishing cause of second crush during surgery, many providers continue to avoid regional anesthesia [1].

\section{Discussion}

Much of the literature evaluating the safety of regional anesthesia in patients with MS is contradictory and sparse. Few studies have been completed and, those that have, have primarily been retrospective chart analyses. Although there is a great need for further investigation on this topic for definitive recommendations, current data suggests that risks previously associated with neuraxial blockade in patients with neurologic disorders may be less frequent than believed in the past [1]. Hebl, Horlocker and Schroeder conducted a retrospective chart review from the Mayo Clinic, spanning over twelve years from 1988 to 2000 [7]. They examined the effects of 139 patients with a history of a central nervous system disorder that received a neuraxial block for a surgical procedure. Prior to neuraxial blockade, motor weakness was the most frequent neurologic deficit among patients. Of the 139 patients, 130 had stable neurologic exams within six months of the block and 74 patients had active symptoms at the time of surgery. Despite over $50 \%$ of patients having active symptoms none of the 139 patients had documentation of postoperative deficits [7]. There were no new or worsening postoperative neurologic deficits found during the follow-up period which averaged sixty days $(0.0 \%$ with a $95 \% \mathrm{CI})$. These results do not support the previous recommendations from Dripps and Vandam which have greatly influenced practice standards for the past fifty years [1]. Additionally, it was previously believed that LA dosing should be lowered in patients with MS and epinephrine additives should be avoided. However, only 11 of the 139 patients received reduced dosing and 72 patients had epinephrine added to their LA mixture and still no postoperative neurologic deficits were noted. Although these results cannot provide definitive recommendations of dosing or use of epinephrine, the study suggests that neuraxial blockade use with standard dosing in the patient with MS should not be 
considered contraindicated as it was previously thought to be The authors summarize that the decision to use neuraxial anesthesia techniques on patients with pre-existing neurologic disorders should be evaluated case by case and a thorough preoperative assessment is crucial. Many patients with advanced deficits affecting the respiratory and cardiovascular systems may benefit from the blunting of the sympathetic nervous system using regional techniques [7].

Ingrosso and colleagues evaluated the effects of peripheral nerve blocks for orthopaedic procedures in two patients with multiple sclerosis. Both patients were females ages 57 and 61 and had been diagnosed with MS for more than twenty years. Each patient had fractures isolated to the tibia and received a Bib lock. A Biblock consists of a standard femoral block combined with a sciatic nerve block to provide regional analgesia to the lower extremity. The blocks were performed identically using peripheral electrical nerve stimulation with insulated needles. The femoral nerve block and the sciatic nerve block each utilized $15 \mathrm{ml} 0.75 \%$ ropivacaine. Both blocks were observed and evaluated to be successful and set up rapidly. Each surgical procedure lasted an average of 75 minutes. The patients were evaluated immediately post operatively and at 30 days post procedure. Neither patient showed signs of exacerbation or progression of the disease at any point during evaluation. Although this study is clearly limited by sample size, it examined two patients at different stages of the disease that both showed positive outcomes and lack of disease progression after receiving a regional block. Before conducting this study, the authors only found 27 bibliographic references on the topic of patients with multiple sclerosis and regional anesthesia, with only 10 of those being in the last 10 years. The authors chose orthopaedic procedures of the lower extremities to avoid providing blockade to any typical central MS lesions to reduce influence of either supplies or drugs on those anatomical structures. The site of surgery allowed a total locoregional approach. Performing this type of block allowed avoidance of medullary block and emotional stress from general anesthesia. The prolonged postoperative analgesia provided from the Bib lock provided a stress-free postoperative course. The authors concluded that peripheral nerve blocks should be considered safe and should be the choice of anesthetic for the patient with MS when surgery allows for it [8].

A case report by Koff and colleagues describes a severe brachial plexopathy after a interscalene nerve block for a total shoulder arthroplasty in a patient with MS [9]. Despite known benefits associated with regional anesthesia in patients undergoing joint replacement, use in the patient with MS is not definitive. Although MS is a disorder of the central nervous system, there is data supporting peripheral involvement in $4-14 \%$ of patients [10]. Postoperatively, the patient reported dense sensory and motor block and was comfortable for the first hour. The next day, the patient was experiencing $5 / 10$ pain with persistent flaccid motor block of his entire right arm. A neurology consults and electro myelogram were ordered. A diagnosis of brachial neuritis was made, and high dose steroids were prescribed. On post-operative day 11, complete paresis of the right arm remained, and new electromyography showed active denervation of all muscles in the arm with no voluntary motor recruitment. At 3 months, the patient showed signs of reinnervation of the muscles of the arm. However, after 8 months, the patient still had significant weakness in the right upper extremity, 50\% loss of motion and visual atrophy of the arm as well as the pectoralis muscles of the right side [10]. Despite testing such as electromyography and MRI, it remains difficult to identify and differentiate an ethology when multiple factors were involved. Potential factors include direct trauma from the nerve block, neurotoxicity from LA, patient positioning such as extreme abduction and external rotation; all which can occur to any patient undergoing total shoulder replacement [11]. An additional confounding variable in diagnosing the cause of postoperative neurologic deficits in MS is that an exacerbation may be caused by many other non-traumatic factors such as hyperthermia, stress, and electrolyte abnormalities. Therefore, although the mechanism of this injury is not definitive, the pre-existing pathology of the peripheral nervous system may have been a contributing factor. Authors conclude that the decision to perform peripheral nerve blocks must be made on a case by case basis, with an understanding that the peripheral nervous system may be somewhat involved in the pathology of MS [9].

\section{Conclusion}

While it is certain that surgical stress negatively impacts the patient with MS, there are no definitive studies on the long term or immediate implications of nerve blocks. Further research is needed in this area, but recent studies show promise for positive outcomes for these patients. Theoretically, regional anesthesia/ analgesia would reduce pain and stress associated with surgery thus reducing the risk of exacerbation. Nonetheless, the theory of double crush phenomenon must be considered. Much of the literature supports the use of peripheral nerve blocks and epidural anesthesia/analgesia and reports that MS should not be considered a contraindication to these pain control modalities. However, spinal anesthesia should still be avoided due to proximity to the CNS and high concentrations of LA to central lesions. The decision to proceed with regional anesthesia in the patient with MS must be made on a case by case basis and careful considerations must be made regarding the risks and benefits. It is important for the anesthesia provider to tightly control temperature and stress during the perioperative period to exclude those as causes of disease exacerbation. Although more research needs to be done for definitive recommendations, overall evidence supports the safety and efficacy of regional anesthesia in patients with MS and presence of the disease should not be considered an absolute contraindication. 


\section{Conflict of Interest}

There is no economic or conflict of interest present by the author

\section{Disclosure}

I have no conflicts of interest

\section{Financial Support and Sponsorship}

\section{None}

\section{References}

1. Jacob AK, Kopp SL (2011) Regional Anesthesia in the Patient with Preexisting Neurologic Disorders. Advances in Anesthesia 29(1): 1-18.

2. McSwain JR, Doty JW, Wilson SH (2014) Regional anesthesia in patients with pre-existing neurologic disease. Current Opinion in Anaesthesiology 27(5): 538-543.

3. Makris A, Piperopoulos A, Karmaniolou I (2014) Multiple sclerosis: basic knowledge and new insights in perioperative management. Journal of Anesthesia 28(2): 267-278.

4. Miller RD (2010) Miller's anesthesia ( $7^{\text {th }}$ Edn.) Philadelphia, PA: Churchill Livingstone/Elsevier.
5. Stoelting RK, Hines RL, Marschall KE (2012) Stoelting's anesthesia and co-existing disease (6 $6^{\text {th }}$ Edn.) Philadelphia: Saunders/Elsevier.

6. Dripps RD, Vandam LD (1956) Exacerbation of pre-existing neurologic disease after spinal anes- thesia. N Engl J Med 255(18): 843-849.

7. Hebl JR, Horlocker TT, Schroeder DR (2006) Neuraxial anesthesia and analgesia in patients with preexisting central nervous system disorders. Anesth Analg 103(1): 223-228.

8. Ingrosso M, Cirillo V, Papasso A, Merolla V, Cecere F (2005) Femoral and sciatic nerves block (BiBlock) in orthopedic traumatologic lower limbs surgery in patients with multiple sclerosis. Minerva Anestesiol 71: 223226.

9. Koff MD, Cohen J, McIntyre J, Carr CF, Sites BD (2008) Severe Brachial Plexopathy after an Ultrasound-guided Single-injection Nerve Block for Total Shoulder Arthroplasty in a Patient with Multiple Sclerosis. Anesthesiology 108(2): 325-328.

10. Misawa S, Kuwabara S, Mori M, Hayakawa S, Sawai S, et al. (2008) Peripheral nerve demyelination in multiple sclerosis. Clinical Neurophysiol 119: 1829-1833.

11. Team BioPlus Health (2016) Anti-Myelin Antibodies in Multiple Sclerosis.
This work is licensed under Creative Commons Attribution 4.0 License

To Submit Your Article Click Here:

Submit Article

DOI: $10.32474 /$ GJAPM.2020.03.000158

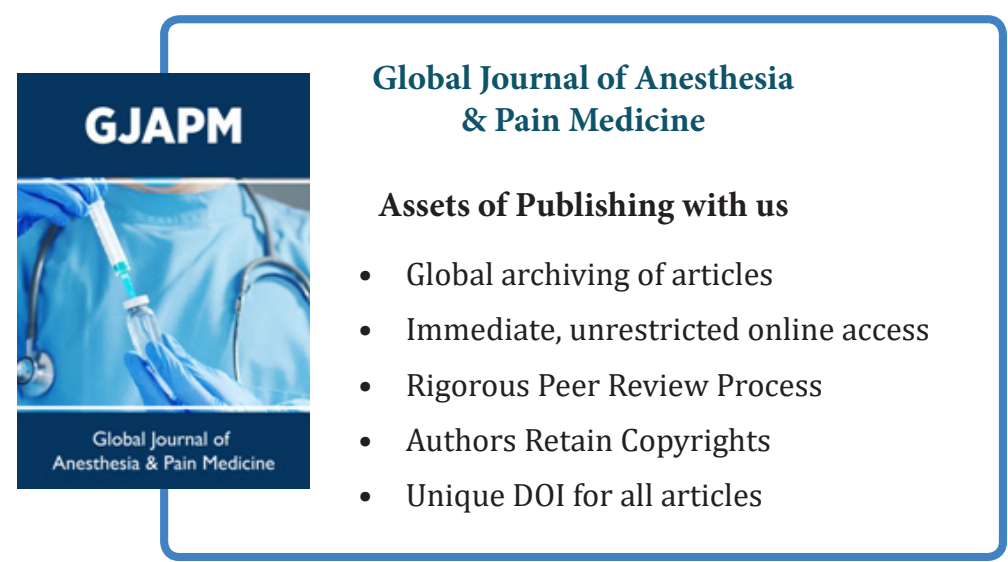

